\title{
Measurement and calculation of neutron leakage spectra from slab samples of beryllium, gallium and tungsten irradiated with 14.8 MeV neutrons
}

\author{
Y.B. Nie ${ }^{1, a}$, X.C. Ruan ${ }^{1}$, J. Ren ${ }^{1}$, S. Zhang ${ }^{2,4}$, R. Han ${ }^{2}$, J. Bao ${ }^{1}$, H.X. Huang ${ }^{1}$, Y.Y. Ding ${ }^{1,3}$, H.C. Wu ${ }^{1}$, P. Liu ${ }^{1}$, \\ and Z.Y. Zhou ${ }^{1}$ \\ ${ }^{1}$ China Nuclear Data Center, China Institute of Atomic Energy, Beijing 102413, China \\ 2 Institute of Modern Physics, Chinese Academy of Sciences, Lanzhou 730000, China \\ 3 School of nuclear science and technology, Lanzhou University, Lanzhou 730000, China \\ ${ }^{4}$ Inner Mongolia University for the Nationalities, Inner Mongolia, Tongliao 028000, China
}

\begin{abstract}
In order to make benchmark validation of the nuclear data for gallium (Ga), tungsten (W) and beryllium $(\mathrm{Be})$ in existing modern evaluated nuclear data files, neutron leakage spectra in the range from 0.8 to $15 \mathrm{MeV}$ from slab samples were measured by time-of-flight technique with a BC501 scintillation detector. The measurements were performed at China Institute of Atomic Energy (CIAE) using a D-T neutron source. The thicknesses of the slabs were 0.5 to 2.5 mean free path for $14.8 \mathrm{MeV}$ neutrons, and the measured angles were chosen to be $60^{\circ}$ and $120^{\circ}$. The measured spectra were compared with those calculated by the continuous energy Monte-Carlo transport code MCNP, using the data from the CENDL-3.1, ENDF/B-VII.1 and JENDL-4.0 nuclear data files, the comparison between the experimental and calculated results show that: The results from all three libraries significantly underestimate the cross section in energy range of $10-13 \mathrm{MeV}$ for $\mathrm{Ga}$; For W, the calculated spectra using data from CENDL-3.1 and JENDL-4.0 libraries show larger discrepancies with the measured ones, especially around $8.5-13.5 \mathrm{MeV}$; and for $\mathrm{Be}$, all the libraries led to underestimation below $3 \mathrm{MeV}$ at $120^{\circ}$.
\end{abstract}

Due to a very low melting point $\left(29.78^{\circ} \mathrm{C}\right)$ and a very high boiling point $\left(2229^{\circ} \mathrm{C}\right)$, Ga becomes an candidate element in Chinese Initiative Accelerator Driven Systems (CIADS) project for liquid-metallic coolant [1]. W is one of the most promising candidate for spallation targets and other structural materials of CIADS project, as well as an important material in fission and fusion devices [2]. Be is another important material in the fission and fusion devices for multiplying neutrons in the core of fission research reactors and in the blankets of Deuteron-Tritium (D-T) fusion reactors [3]. The accuracy of nuclear data files for $\mathrm{Ga}, \mathrm{W}$ and $\mathrm{Be}$ is very important for nuclear device design. The purpose of this work is to validate currently available modern nuclear data files, especially the CENDL-3.1 file, by carrying out benchmark experiment

\section{Experiments and calculations}

\subsection{Experiments}

The measurements were performed by using the benchmark experimental facility at China Institute of Atomic Energy (CIAE). A schematic view of the experimental arrangement is shown in Fig. 1, which was described in detail earlier in Reference [4].

The $\mathrm{T}(\mathrm{d}, \mathrm{n})^{4} \mathrm{He}$ reaction served as fusion neutron source. The D+ beam was bunched about $1.5 \mathrm{~ns}$ in Full Width at Half Maximum (FWHM) and the repetition

a e-mail: nieyb@163.com rate was $1.5 \mathrm{MHz}$. The average beam current and beam energy were about $30 \mu \mathrm{A}$ and $300 \mathrm{keV}$, respectively. An air-cooling device was used for cooling the target. For getting the time structure of the pulsed source neutrons, a stilbene scintillation crystal (monitor) of $5.08 \mathrm{~cm}$ in diameter and $2.54 \mathrm{~cm}$ in length was placed at about $800 \mathrm{~cm}$ from the Ti-T target at $0^{\circ}$. A silicon surface barrier (SSD) detector, positioned at $135^{\circ}$ with respect to the $\mathrm{D}^{+}$beam, was used to monitor the neutron yields by counting the associated ${ }^{4} \mathrm{He}$ particles. All the samples used for the experiment have purity better than $99.3 \%$. For detecting the leakage neutrons from samples, a BC501A liquid scintillator detector, which was located behind the concrete wall with a collimated hole, with a size of $5.08 \mathrm{~cm}$ in diameter and $2.54 \mathrm{~cm}$ in length was used.

The leakage neutron spectra were obtained from the results of the sample-in measurements by subtracting those of sample-out measurements, and the results were normalized by the number of source neutrons. The uncertainties combine the statistical and systematic uncertainties. The systematic uncertainties were mainly caused by the neutron detection efficiency $(\leq 3 \%)$, the source neutron yield $(\leq 3 \%)$ and the scattering angle ambiguity $(\leq 1 \%)$.

\subsection{Monte Carlo calculations}

The theoretical calculation was performed using a threedimensional continuous energy Monte Carlo transport code, MCNP-4B. The evaluated nuclear data of $\mathrm{Ga}, \mathrm{W}$ and

(c) The Authors, published by EDP Sciences. This is an Open Access article distributed under the terms of the Creative Commons Attribution License 4.0 (http://creativecommons.org/licenses/by/4.0/). 


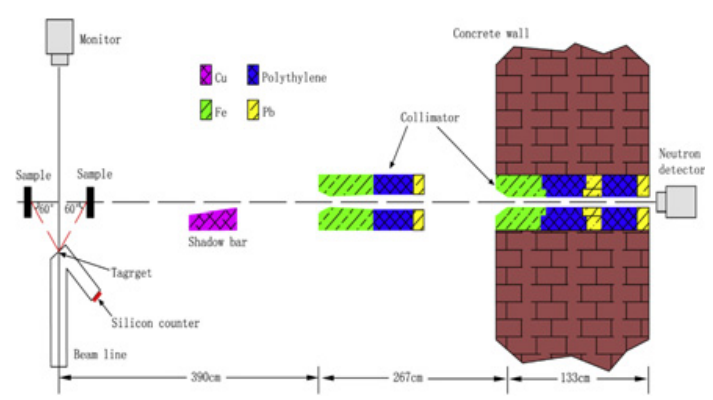

Figure 1. Lay-out of experiment for measuring the neutron leakage spectra from slab sample.

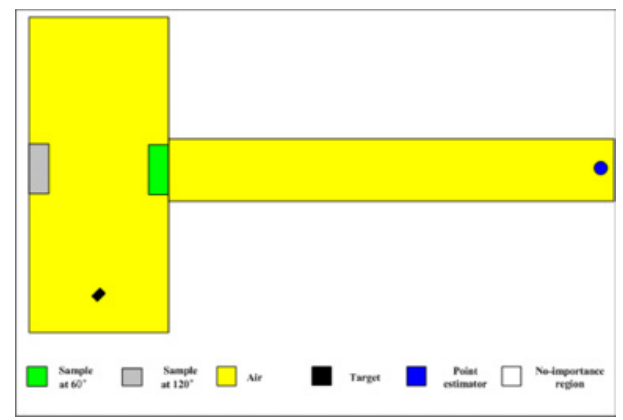

Figure 2. Model for the MCNP calculations.

Be were taken from CENDL-3.1 [5], ENDF/B-VII.1 [6] and JENDL-4.0 [7], the data of other materials were all taken from the ENDF/B-VII.1. A point detector estimator was used to tally the leakage neutron time of flight spectra for comparing with the measured ones. The calculations for two angles of about $60^{\circ}$ and $120^{\circ}$ were performed with the model shown in Fig. 2. The left sample was filled with air when the simulation was performed for $60^{\circ}$, while the right sample was filled with air for $120^{\circ}$ simulation. For the background simulation, both samples were filled with air. The neutron histories adopted were $10^{9}$ and the statistical uncertainties of each time bin were smaller than $1 \%$.

In the MCNP simulations, the detailed experimental parameters were taken into account. These included the neutron energy distribution produced on the thick target, the angle dependent energy distribution of the source neutrons, the neutron detection efficiency, and the time structure of the pulsed beam (TME). Both of the angular distribution and angle dependent energy distribution of the source neutrons were calculated by the TARGET code [8]. The value of TME variable was defined by using experimentally measured time of flight spectra of source neutrons by the monitor.

\section{Results and discussion}

\section{1. $\mathrm{Ga}$}

The neutron leakage spectra for Ga calculated with the evaluated data files comparing with the measured ones at $60^{\circ}$ and $120^{\circ}$ are shown in Fig. 3. From the comparison, we can conclude that:

1. In the $13-15 \mathrm{MeV}$ neutron energy range, a sharp elastic peak is observed. All the calculated results using these three libraries have large discrepancies with the experimental ones.

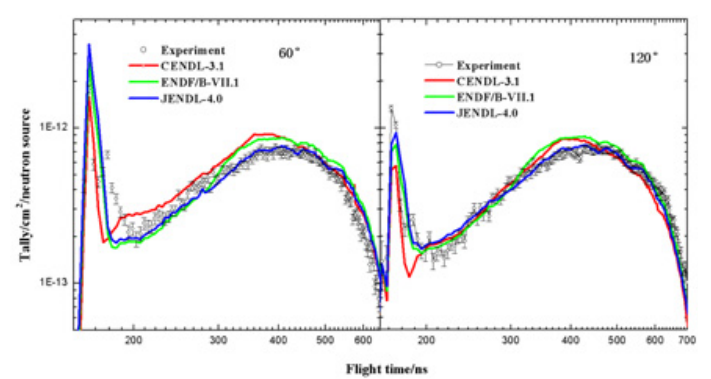

Figure 3. Comparison of experimental and calculated neutron leakage spectra from ${ }^{\text {nat }} \mathrm{Ga}$ slab with $143 \mathrm{keV}$ electron equivalent threshold (6.4 cm thickness).

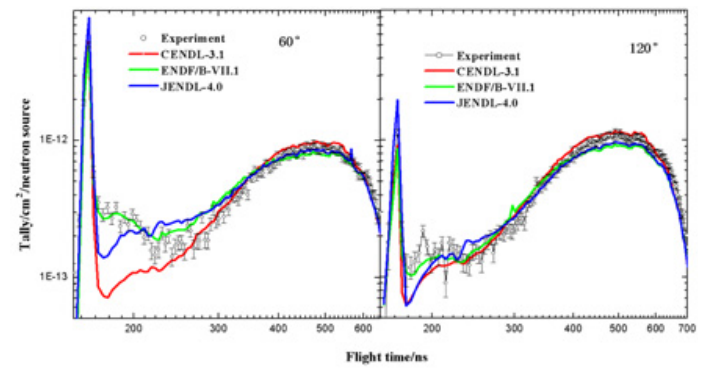

Figure 4. Comparison of experimental and calculated neutron leakage spectra from ${ }^{\text {nat }} \mathrm{W}$ slab with $143 \mathrm{keV}$ electron equivalent threshold ( $7.35 \mathrm{~cm}$ thickness).

2. In the $10-13 \mathrm{MeV}$ neutron energy range, a small, but clear peak is observed. The contribution originates from discrete level of inelastic scatterings $\left(\left(\mathrm{n}, \mathrm{n}^{\prime}\right) \mathrm{d}\right)$ and all MCNP simulations using the three libraries do not show a peak as the experimental data do.

3. In the $3-10 \mathrm{MeV}$ neutron energy range, the contribution comes from the continuous level of inelastic scattering $\left(\left(\mathrm{n}, \mathrm{n}^{\prime}\right) \mathrm{C}\right)$. For $60^{\circ}$, the calculated spectra with the CENDL-3.1 are overestimated about $15 \%$. For $120^{\circ}$, all the calculated spectra agree well with the experiment.

4. In the $0.8-3 \mathrm{MeV}$ neutron energy range, in which most of the contribution comes from the $(n, 2 n)$ reaction channel. The calculated spectra with the ENDF/B-VII. 1 are overestimated about $20 \%$ at $60^{\circ}$ and $10 \%$ at $120^{\circ}$.

\section{2. $\mathrm{W}$}

The neutron leakage spectra from $\mathrm{W}$ calculated with the evaluated data files comparing with the measured ones at $60^{\circ}$ and $120^{\circ}$ are shown in Fig. 4. From these results, the following observations are made:

1. In the $12.5-15 \mathrm{MeV}$ neutron energy range, the major contribution comes from the elastic scattering. The calculated elastic scattering peak with the evaluated data of the JENDL-4.0 libraries is higher than the experimental one, while those of the ENDF/B-VII.1 and CENDL-3.1 libraries are underestimated.

2. In the 7-12.5 MeV neutron energy range, the calculated spectra with the ENDF/B-VII.1 give agreement with the experimental ones at $60^{\circ}$ and $120^{\circ}$, but those with the JENDL-4.0 and CENDL3.1 are largely underestimated. 


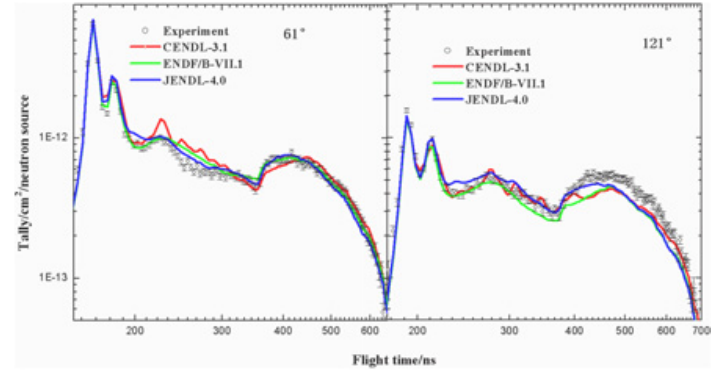

Figure 5. Comparison of experimental and calculated neutron leakage spectra from Be slab with $143 \mathrm{keV}$ electron equivalent threshold (11 cm thickness).

3. In the $3-7 \mathrm{MeV}$ neutron energy range, the results from the ENDF/B-VII.1 and JENDL-4.0 show overestimation at $60^{\circ}$.

4. Below $3 \mathrm{MeV}$, the calculated spectra with the ENDF/B-VII.1 and JENDL-4.0 libraries reproduce the experimental ones, while those with the CENDL-3.1 library cause overestimation at $60^{\circ}$. At $120^{\circ}$, the results from the CENDL-3.1 give agreement with the experimental ones, while those with the JENDL-4.0 and ENDF/B-VII.1 are underestimated.

\section{3. $\mathrm{Be}$}

The neutron leakage spectra calculated with the evaluated data files comparing with the measured ones at $61^{\circ}$ and $121^{\circ}$ are shown in Fig. 5. From the comparison, we can conclude that:

1. In the $12-15 \mathrm{MeV}$ range at $61^{\circ}$, and $9.5-13 \mathrm{MeV}$ range at $121^{\circ}$, the calculated neutron spectra with all libraries agree well with the experiment.

2. In the $2.8-12 \mathrm{MeV}$ range at $61^{\circ}$, most contribution comes from the $(n, 2 n)$ reaction, the calculated spectra with the CENDL-3.1 show seriously overestimation.

3. In the $0.8-2.8 \mathrm{MeV}$ range at $121^{\circ}$, all the calculated neutron spectra show a significant underestimation, around 20\%, especially the ENDF/B-VII.1.

\section{Discussion}

In order to find the source of these discrepancies between the calculated results and the experimental ones, the reaction cross sections, the secondary neutron energy spectra and the cross section angular distributions from the CENDL-3.1, ENDF/B-VII.1 and JENDL-4.0 nuclear data files were compared.

For $\mathrm{Ga}$, the values of $\left(\mathrm{n}, \mathrm{n}^{\prime}\right) \mathrm{d}$ cross section and the elastic scattering angular distribution should be improved in all libraries. The $\left(\mathrm{n}, \mathrm{n}^{\prime}\right) \mathrm{C}$ cross section, as shown in Table 1 , the value is much higher for ${ }^{69} \mathrm{Ga}$ in the CENDL-3.1, which causes the CENDL-3.1 overestimated in the 3-10 MeV energy range.

For W, as shown in Fig. 6, the energy distribution of $\left(n, n^{\prime}\right) C$ reaction in the CENDL-3.1 is significantly lower than that in the ENDF/B-VII.1 from 7 to $13 \mathrm{MeV}$, which cause the CENDL-3.1 underestimated at this energy range. The contributions of the $(n, 2 n)$ reaction
Table 1. The values of $\left(n, n^{\prime}\right) C$ cross section for Ga from different nuclear data files $(\mathrm{En}=14.5 \mathrm{MeV})$.

\begin{tabular}{cccc}
\hline & CENDL-3.1 & ENDF/B-VII.1 & JENDL-4.0 \\
\hline${ }^{69} \mathrm{Ga}$ & 0.586 & 0.492 & 0.492 \\
\hline${ }^{71} \mathrm{Ga}$ & 0.603 & 0.610 & 0.498 \\
\hline${ }^{\text {nat } \mathrm{Ga}}$ & 0.593 & 0.539 & 0.495 \\
\hline
\end{tabular}

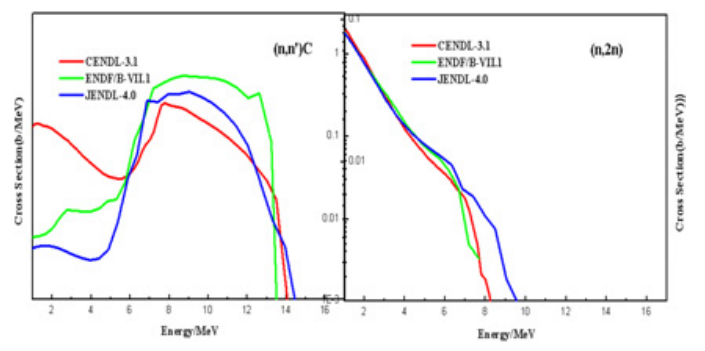

Figure 6. The energy distribution from $\left(\mathrm{n}, \mathrm{n}^{\prime}\right) \mathrm{C}$ reactions and $(n, 2 n)$ reactions of ${ }^{\text {nat }} \mathrm{W}(\mathrm{En}=14.5 \mathrm{MeV})$.

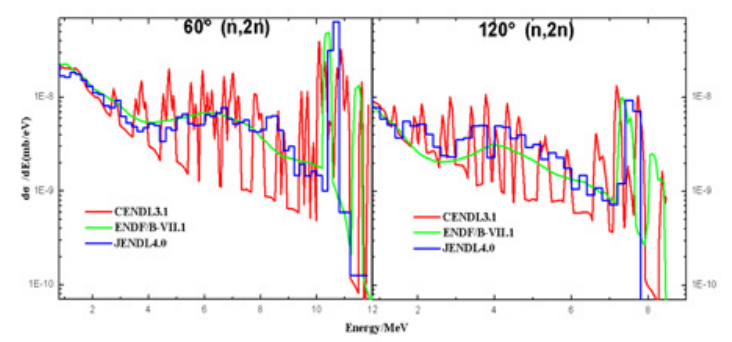

Figure 7. The neutron spectra of $(n, 2 n)$ reactions at $60^{\circ}$ and $120^{\circ}$ $(\mathrm{En}=14.5 \mathrm{MeV})$.

in the ENDF/B-VII.1 and JENDL-4.0 are considerably higher than CENDL-3.1 from 3 to $7 \mathrm{MeV}$, which cause the ENDF/B-VII.1 and JENDL-4.0 overestimated at this energy range.

For $\mathrm{Be}$, the secondary neutron emission spectra are mainly contributed by the $(n, 2 n)$ reaction except elastic scattering. As shown in Fig. 7, the neutron energy spectra of the $(n, 2 n)$ reactions those retrieved from the CENDL3.1 and JENDL4.0 are quite different. The double differential cross section data of CENDL-3.1 are treated as many inelastic scattering peaks overlap with a continuum part. Therefore many peaks can be seen in the calculated spectra.

\section{Summary}

Benchmark experiments to validate the existing evaluated nuclear data files for $\mathrm{Ga}, \mathrm{W}$ and $\mathrm{Be}$ were conducted through time-of-flight spectra measurement in the energy range between 0.8 and $15 \mathrm{MeV}$. The main purpose of these experiments was to check the evaluated nuclear data in the CENDL-3.1 file. Through the experiment, we can conclude that: the elastic scattering angular distribution, the $\left(n, n^{\prime}\right) d$ cross section and $\left(n, n^{\prime}\right) C$ cross section for $\mathrm{Ga}$, the energy distribution of $\left(n, n^{\prime}\right) \mathrm{C}$ reaction, the $\left(n, n^{\prime}\right) d$ cross section and $\left(n, n^{\prime}\right) C$ cross section for $W$, the energy distribution of $(n, 2 n)$ reaction for Be, these data in the CENDL-3.1 should be improved. 
The authors gratefully acknowledge H. Chen, F. Zhao and $\mathrm{K}$. Zhang, for their excellent operation of the D-T neutron source at CIAE.

\section{References}

[1] N. Afroze, M.S. Uddin, et al. NIM(B) 336, 1 (2014)

[2] H. Kishimoto, T. Shibayama et al. J. Nucl. Mat. 417, 387 (2011)

[3] M. Nakamichi, J. Kim. Fus. Eng. Des. 89, 1304 (2014)
[4] Y. Nie, J. Ren, et al. Fus. Eng. Des. 105, 8 (2016)

[5] Z. Ge, Z. Zhao, et al. Korean Phys. Soc. 59, 1052 (2011)

[6] M. Chadwick, M. Herman, et al. Nucl. Data Sheets 112, 2887 (2011)

[7] K. Shibata, O. Iwamoto, et al. J. Nucl.Sci. Technol. 48, 1 (2011)

[8] D. Schlegel, TARGET User's Manual, Laborbericht PTB-6.42-05-2. Braunschweig, 2005, Germany 